\title{
Research Progress and the Internal Essence of the Petroleum Reservoirs Percolation Mechanics
}

\author{
Wei Gong1,2, Qinfeng Di1,2*, Renliang Zhang1,2, Xinliang Wang1,2, Weipeng Ding ${ }^{1,2}$ \\ ${ }^{1}$ Shanghai Institute of Applied Mathematics and Mechanics, Shanghai University, Shanghai \\ ${ }^{2}$ Shanghai Key Laboratory of Mechanics in Energy Engineering, Shanghai University, Shanghai \\ Email: "qinfengd@sina.com \\ Published on Advances in Porous Flow, Vol. 3, No. 1, 2013; available on \\ http://www.hanspub.org/journal/PaperInformation.aspx?paperlD=9678
}

\begin{abstract}
Percolation mechanics is the science that deals with fluid flow in porous media, which has wide applications in many aspects. Due to a variety of changing objects, there are various and complicated percolation mechanics problems and related models in the petroleum engineering. In this work, the authors analyzed various percolation mechanics problems, discussed the mechanical mechanism and the basic models of percolation mechanics in different reservoir media, and reviewed the percolation mechanics essence of the effective methods for oil discovery such as polymer flooding, microbial enhanced oil recovery. The results showed that no matter under what technical environments, the laws of percolation could be described as the modified models of percolation mechanical fundamental equation under special circumstance. This conclusion provides an important reference for the study of percolation mechanical models on new subjects.
\end{abstract}

\section{Keywords}

Petroleum Reservoirs, Seepage Models, Internal Essence, Porous Media, Fluid

"Corresponding author. 